\title{
ESTABILIDAD FENOTÍPICA DE GENOTIPOS DE ALGODÓN (Gossypium hirsutum L.) EN EL CARIBE COLOMBIANO
}

\author{
FENOTYPIC STABILITY OF COTTON \\ (Gossypium hirsutum L.) GENOTYPES \\ IN THE COLOMBIAN CARIBBEAN
}

Hermes Araméndiz T. ${ }^{1}$ Miguel Espitia C. ${ }^{2}$ Alejandro Agámez C. ${ }^{3}$

Carlos Cardona A. ${ }^{4}$ Juana Robles $\mathrm{G} .^{1}$

\section{RESUMEN}

El algodón es el principal cultivo durante el segundo semestre del año en la región caribe. Es desarrollado en condiciones ambientales y tecnológicas variables que afectan los rendimientos de fibra de los cultivares y la competitividad del sistema de producción. El objetivo de esta investigación fue analizar la estabilidad fenotípica del rendimiento de fibra de diez genotipos de algodón en ocho ambientes de la Costa Atlántica. Se utilizó el diseño de bloques completos al azar con cuatro repeticiones en todos los ambientes. Para los análisis de estabilidad, se utilizaron las metodologías propuestas por Lin $\mathcal{E}$ Binns (1988a) y Carneiro (1998). El análisis de variancia combinado detectó interacción genotipo-ambiente significativa $(p<0.01)$, como reflejo en el cambio de la superioridad de los cultivares de un ambiente a otro. De acuerdo con la metodología empleada, los cultivares L. Cesar-151 y Delta opal resultaron ser los

${ }^{1}$ Docente. Universidad de Córdoba. Montería. Carrera 6ํ76103. haramendiz@hotmail.com

2 Docente. Universidad de Córdoba. Mespitia37@hotmail.com

${ }^{3}$ Ingeniero Agrónomo. Corpoica. C.I. Turipana. alejandro_ agamez@yahoo.com

4 Docente. Universidad de Córdoba. ccardona@sinu.unicordoba.edu.co

5 Docente. Universidad de Córdoba. jrobles@sinu.unicordoba. edu.co más deseables por presentar los menores valores de Pig (más estables) y los mayores rendimiento promedios de fibra. La metodología de Carneiro (1998) seleccionó, por su mayor estabilidad fenotípica y rendimientos, a L. Cesar-151 y Delta Opal y discriminó, para ambientes favorables, y a L. Cesar-149 y Corpoica M-123 para ambientes desfavorables.

Palabras clave: Interacción genotipo-ambiente, rendimiento de fibra, parámetros de estabilidad.

\section{SUMMARY}

Cotton is the main crop of the second term of the year in the Colombian Northern Coast. It grows under variable environmental and technological conditions that affect cultivar fiber yield and the competitiveness of the production system. The main objective of this research was to analyze the phenotypic stability of the fiber yield of ten cotton genotypes in eight environments of the Atlantic Coast. A complete randomized block design with four replicates was used in all experiments. The stability analysis was made by means of the methodologies proposed by Lin E Binns (1988) and Carneiro (1998). The combined variance analysis detected significant interaction genotype-environment $(p<0.01)$, as reflected in the change of the superiority of genotypes from an environment to another. According to the methodology employed, the cultivars L. Cesar -151 and Delta opal 
showed the best results, since they presented the lowest Pig values (more stable) and the higher average fiber yield. The methodology of Carneiro (1998) selected, due to greater phenotypic stability and yields, L. Cesar-151 and Delta Opal for better environmental conditions and L. Cesar-149 and Corpoica M-123 for less favorable environments.

Key words: Interaction genotype-environment, fibre yield, parameters of stability.

\section{INTRODUCCIÓN}

El algodón de fibra media es la principal especie agrícola cultivada en el segundo semestre del año en el caribe colombiano; genera el $6 \%$ de los empleos directos entre los cultivos transitorios. El área sembrada en el 2006 fue de 35.000 hectáreas, las cuales representaron el $65 \%$ de la producción nacional, con rendimientos de algodón semilla promedios de $2.000 \mathrm{~kg} \mathrm{ha}^{-1} \mathrm{y}$ rendimientos de fibra promedio de $863 \mathrm{~kg} \mathrm{ha}^{-1}$; guarismo éste que permitió al país ubicarse en el puesto 15, a nivel mundial (Arias, 2007). Esta actividad es realizada en condiciones ambientales y tecnológicas variables, sin riego suplementario, con uso de cultivares foráneos y, muchas veces, de poca adaptación a las condiciones cambiantes del trópico. Estos factores, unidos a otros de origen político y económico nacional e internacional, han afectado la competitividad del cultivo. Ello ha conllevado a la reducción del área de siembra de 250.000ha en la cosecha 1990-1991 a 51.225ha en la temporada 20062007 (Mendoza et al. 1999; Arias, 2007), conduciendo a la conversión del país de exportador a importador de fibra y, con ello, se ha originado un vacío en la generación de empleos directos e indirectos en esta actividad (Espinal et al. 2005).

La etapa final de cualquier programa de mejoramiento genético exige la realización de experimentos en ambientes divergentes, en razón a que los productores realizan sus cultivos en condiciones muy complejas, con un gran efecto ambiental en la expresión del genotipo y cambios en la expresión del fenotipo (Kang, 1998). Por lo anterior es necesario, estimar el potencial de rendimiento, al igual que la adaptabilidad y la estabilidad fenotípica de cultivares avanzados en esas ofertas ambientales y de manejo agronómico, de igual manera obtener conclusiones más confiables de las líneas en evaluación y así realizar recomendaciones mucho más precisas a esas condiciones impredecibles y acordes con las particularidades de las zonas productoras de la región caribe, especialmente, las concerniente con el régimen de lluvia.

La evaluación de cultivares en los ambientes no arroja una respuesta sencilla, por lo general, presentan una interacción con el mismo, definida como una respuesta diferencial de los cultivares a la variación de los ambientes, dificultando la selección de genotipos adaptados. Así mismo, influye esta interacción en la eficiencia de la estimación de parámetros genéticos, progresos con la selección y un reducido progreso de los programas de mejoramiento (Duarte $\mathcal{E}$ Vencovsky, 1999). Para regiones con irregularidad entre localidades y años en la oferta ambiental, como el caribe colombiano y progresos en los rendimientos de la agricultura son posibles con la identificación de genotipos que expresen su potencial genético en ambientes más favorables y específicos, de tal manera, que ello conduzca a un menor costo de la producción de cada kilo de fibra.

Chloupek E Hrstkova (2005) indican que la adaptabilidad es el resultado de la interacción del genotipo con el ambiente y esta característica esta controlada genéticamente, ya que QTL, asociados con la adaptabilidad, han sido identificados en trigo y cebada (Kato et al. 2000; Snape et al. 2007), posibilitando la habilidad de explorar atributos ambientales y agronómicos. El ambiente comprende factores naturales, como la geografía, los años, el suelo y la precipitación que es el más impredecible por su variación de año en año, además de factores humanos, como las prácticas agronómicas (Lin E Binns, 1988b; Dencic et al. 2000; Chloupek et al. 2004).

Para entregar a productores o recomendar cualquier cultivar es fundamental la realización de estudios de adaptabilidad y de estabilidad fenotípica. Existen muchos métodos que han venido siendo aplicados para analizar la interacción genotipo - ambiente. Entre ellos, sobresalen los que se basan en el análisis de regresión (Finlay $\mathcal{E}$ Wilkinson, 1963; Eberhart $\mathcal{E}$ Russell, 1966; Verma et al. 1978); análisis multivariados (Lin \& Thompson, 1975; Lin E Butler, 1990); análisis de componentes principales (Kroonenberg \& Basford, 1989; Gauch, 1988; Gauch E Furnas, 1991); componentes de variancia (Plaisted, 1960; 
Wricke, 1962; Shukla, 1972) y análisis no paramétrico (Lin E Binns, 1988a; Carneiro, 1998; Abeysiriwaderna, 2001), entre otros.

La escogencia del método depende de los datos experimentales, principalmente, los relacionados con el número de ambientes disponibles, precisión requerida para la discriminación genotípica y tipo de información deseada. Se enfatizan que algunos métodos son alternativos, en tanto que otros son complementarios, pudiendo ser utilizados conjuntamente en el mejoramiento de plantas (Cruz \& Regazzi, 1997).

La metodología de Lin $\mathcal{E}$ Binns (1988a) es una buena alternativa para la evaluación de cultivares con relación a la interacción genotipo-ambiente. Sobre este método, Huehn (1990), Atroch et al. (2000) y Scapim et al. (2000) señalan que no tiene las limitaciones de la regresión cuya interpretación es más difícil, es simple y fácil de aplicar y caracteriza los genotipos en un solo parámetro (Pi) que asocia la estabilidad con el rendimiento y, que a menor valor, mayor será su estabilidad. Además, la inclusión o retirada de uno o pocos cultivares no causa grandes variaciones en la estimación de los parámetros y permite la clasificación de los genotipos para selección en el proceso de mejoramiento. Este método fue modificado por Carneiro (1998) para identificar los genotipos superiores en ambientes favorables y desfavorables. Estos métodos han sido aplicados en arroz por Abeysiriwaderna (2001) y por Atroch et al. (2000); en maíz por Scapim et al. (2000) y por Murakami et al. (2004); en fríjol por Morais et al. (2001); en quinua por Jacobsen et al. (1996); en alfalfa por Ferreira et al. (2004) y en algodón por Espítia et al. (1993) y por Vertel et al. (1999).

El programa de mejoramiento de algodón de la Corporación Colombiana de Investigación Agropecuaria (CORPOICA) trabaja en áreas tropicales donde existe una alta diversidad ambiental y de manejo agronómico que contribuyen significativamente a la presencia de interacción genotipo - ambiente. Por lo tanto, el objetivo fue conocer la estabilidad fenotípica de líneas avanzadas y variedades comerciales de algodón de fibra media, para seleccionar los mejores genotipos, para las áreas productoras de la región Caribe.

\section{MATERIALES Y MÉTODOS}

Para el presente estudio, se utilizaron los datos de rendimiento de fibra, obtenidos de experimentos realizados en fincas de productores y de centros de investigación de diferentes regiones algodoneras del caribe colombiano, realizados en la cosecha 20032004, bajo la responsabilidad de CORPOICA, en los departamentos de Córdoba, de Cesar y de la Guajira.

Los experimentos fueron sembrados de acuerdo a la época de siembra de cada ambiente, en los municipios de Maicao, El Copey, Codazzi, San Juan del Cesar, Cereté, San Pelayo, Cotorra y Aguachica, utilizando el diseño experimental de bloques completamente al azar, con 10 tratamientos (Línea Cesar -149, Línea Cesar -151, Línea Cesar -153, Línea Turipaná-1, Línea Turipaná-2, Línea Turipaná-3, Línea Turipaná-4, Corpoica M-123, Delta Opal y Sinuana M-137) y cuatro repeticiones. El manejo agronómico utilizado en la investigación fue el mismo que aplicaron los productores en sus lotes comerciales donde estuvieron ubicados cada uno de los experimentos. Las unidades experimentales estuvieron conformadas por seis surcos de $10 \mathrm{~m}$ de longitud, separados a $0,90 \mathrm{~m}$ y con distancias entre plantas de $0,40 \mathrm{~m}$. Las variables consideradas en la investigación fueron rendimiento de fibra, número de motas por planta, porcentaje de fibra, peso de mota y calidad de fibra.

Se realizaron análisis individuales de variancia, seguidos de análisis combinados (conjunto) para identificar la importancia de los efectos de los componentes experimentales del modelo mixto asumido, considerándose como fijo los genotipos y aleatorio las localidades, utilizando el programa SAS (SAS Institute, 2002).

En el método de Lin E Binns (1988a), el desempeño general de los genotipos es definido como el cuadrado medio de la distancia entre la media del cultivar y la respuesta media máxima para todas las localidades, de tal modo que los genotipos, con menores valores, corresponden a los de mejor desempeño. De acuerdo con esta definición, el estimador Pig es determinado por la siguiente formula:

$$
\operatorname{Pig}=\frac{\sum_{j=1}^{n}\left(X_{i j}-M_{j}\right)^{2}}{2 n}
$$


donde:

Pig $=$ estadístico del parámetro de estabilidad del cultivar i;

$\mathrm{X}_{i j}=$ rendimiento de fibra $\left(\mathrm{kg} \mathrm{ha}^{-1}\right)$ del $\mathrm{i}$ - ésimo cultivar en el $\mathrm{j}$ - ésimo ambiente;

$M_{j}=$ respuesta máxima observada entre todos los cultivares en el ambiente j;

$\mathrm{n}=$ número de ambientes.

Carneiro (1998) descompuso el estadístico Pig, con el fin de identificar los genotipos superiores en ambientes favorables y desfavorables, utilizando la misma metodología de clasificación de ambientes de Eberhart $\mathcal{E}$ Russell (1966). En esta descomposición, los siguientes estadísticos son estimados:

Pif $=\frac{\sum_{j=1}^{f}\left(X_{i j}-M_{j}\right)^{2}}{2 f}$ y Pid $=\frac{\sum_{j=1}^{d}\left(X_{i j}-M_{j}\right)^{2}}{2 d}$

donde:

Pif = estadístico de Pig para ambientes favorables;

Pid = estadístico de Pig para ambientes desfavorables;

$f \quad=$ número de ambientes favorables;

$\mathrm{d}=$ número de ambientes desfavorables;

Xij y Mj como fueron definidos anteriormente.

De esta manera, la selección de los cultivares puede ser realizada para los diferentes tipos de ambientes (general y específica), siendo mucho más completa que la propuesta original.

\section{RESULTADOS Y DISCUSIÓN}

Los resultados del análisis de variancia combinado, se encuentran consignados en la Tabla 1 . En ella, se puede apreciar diferencias altamente significativas para todas las fuentes de variación, indicando que los cultivares poseen divergencia en cuanto a su potencial de rendimiento de fibra y que los ambientes satisfacen el pre-requisito de contraste entre las localidades.

De igual manera, la existencia de interacción genotipoambiente (Cultivares x Localidades), denota la dificultad de recomendar, de forma general, cultivares para la región en estudio, por lo que es necesario un estudio más detallado de la interacción genotipo-ambiente para realizar recomendaciones altamente precisas de los cultivares sometidos en estudio. Esta variabilidad de respuesta ha sido divulgada a nivel de especies y de cultivares por Souza $E$ Holanda, 1993; Banziger $\mathcal{E}$ Cooper, 2001 y Chloupek E Hrstkova, 2005.

En la Tabla 2, se observa una amplia variación en el estadístico Pig para los cultivares estudiados en la diferentes localidades, oscilando dichos guarismos entre 4783,19 (L. Cesar - 151) y 96446,44 (L. Turipaná - 3) y el rendimiento de fibra por hectárea promedio, entre 1110 (L. Turipaná - 3) y $1461 \mathrm{~kg} \mathrm{ha}^{-1}$ (L.Cesar - 151). Los cultivares L.Cesar -151 y Delta Opal, registraron los menores valores de Pig con 4783,19 y 6604,51, siendo calificados como los más estables, ya que a menor Pig, pequeño será también el desvío en torno de la producción máxima de cada ambiente e integran el grupo más productivo en los ambientes considerados,

Tabla 1. Análisis de variancia combinado para el rendimiento de fibra $\left(\mathrm{kg} / \mathrm{ha}^{-1}\right)$ de diez cultivares de algodón, evaluados en el caribe colombiano, durante la cosecha 2003-2004.

\begin{tabular}{|l|l|l|l|l|l|}
\hline Fuente de variación & GL & S. de C & C. M. & F & Pr> F \\
\hline Repeticiones (Localidades) & 24 & 6383319 & 265972 & 7,40 & $<.0001$ \\
\hline Cultivares & 9 & 3518078 & 390898 & 6,86 & $<.0001$ \\
\hline Localidades & 7 & 51478809 & 7354116 & 25,62 & $<.0001$ \\
\hline Cultivares x Localidades & 63 & 3588301 & 56957 & 1,59 & 0.0082 \\
\hline Error residual & 216 & 7760511 & 35928 & - & - \\
\hline
\end{tabular}

G.L. : Grados de libertad; S. de C.: Suma de cuadrados; C.M.: Cuadrados medios 
puesto que su rendimiento de fibra fue de 1461 y $1379 \mathrm{~kg}$ ha $^{-1}$, respectivamente. Lo que posiblemente obedezca a la presencia de bloques de genes asociados con la adaptabilidad y la estabilidad, como lo reportaron en otras especies Kato et al. (2000) y Snape et al. (2007) o una mayor estabilidad agronómica (Lin et al. 1986; Piepho, 1996), debido a que registraron un mayor potencial productivo en los ambientes donde fueron evaluados. Ante estos resultados, la línea L. Cesar -151, se convierte en un genotipo promisorio para las zonas algodoneras del caribe colombiano, por lo que puede ser elevado a la categoría de variedad. Igual conducta con la variedad Delta Opal, que puede ser recomendada a los productores por su excelente comportamiento agronómico, como se puede apreciar en la Tabla 3, ya que la característica porcentaje de fibra del genotipo colombiano es superior en 2 y $3 \%$, respecto a las variedades comerciales, lo cual redunda en un mayor rendimiento de fibra. Así mismo,

Tabla 2. Rendimiento medio de fibra $\left(\mathrm{kg} / \mathrm{ha}^{-1}\right)$, parámetros estabilidad para condiciones generales $\left(\mathbf{P}_{\text {ig }}\right)$, condiciones ambientales favorables $\left(\mathbf{P}_{\text {if }}\right)$ y desfavorables $\left(\mathbf{P}_{\text {id }}\right)$, con su clasificación de acuerdo a la metodología de LIN \& BINNS (1988a) y CARNEIRO (1998).

\begin{tabular}{|c|c|c|c|c|c|c|c|}
\hline Cultivares & Media & $\boldsymbol{P}_{\text {ig }}$ & Clasif. & $\boldsymbol{P}_{\text {if }}$ & Clasif. & $\boldsymbol{P}_{i d}$ & Clasif. \\
\hline LC - 149 & 1329 & 19189,22 & 3 & 28040,44 & 5 & 6103,99 & 1 \\
\hline LC - 151 & 1461 & 4783,59 & 1 & 68,43 & 1 & 12642,19 & 5 \\
\hline LC - 153 & 1236 & 57927,86 & 7 & 80054,94 & 9 & 21049,41 & 7 \\
\hline L T - 1 & 1188 & 58313,53 & 8 & 74317,90 & 7 & 31639,58 & 8 \\
\hline L T - 2 & 1125 & 80310,93 & 9 & 102076,88 & 10 & 44034,35 & 10 \\
\hline L T - 3 & 1110 & 96446,64 & 10 & 16291,93 & 3 & 35658,70 & 9 \\
\hline L T - 4 & 1231 & 49603,16 & 5 & 68532,78 & 6 & 18053,79 & 6 \\
\hline CM - 123 & 1257 & 52345,05 & 6 & 78523,43 & 8 & 8714,42 & 2 \\
\hline Delta opal & 1379 & 6604,51 & 2 & 4904,0 & 2 & 9438,54 & 3 \\
\hline SM - 137 & 1316 & 20641,62 & 4 & 26282,41 & 4 & 11240,29 & 4 \\
\hline Media general & 1263 & & & & & & \\
\hline
\end{tabular}

Tabla 3. Valores promedios de rendimiento de algodón -semilla y características de la fibra de los cultivares evaluados en ocho ambientes de la región caribe, 2003-2004.

\begin{tabular}{|l|c|c|c|c|c|c|c|c|}
\hline \multicolumn{1}{|c|}{ Cultivares } & $\begin{array}{c}\text { Alg-Sem } \\
\text { (kg/ha) }\end{array}$ & Fibra \% & $\begin{array}{c}\text { Rendim.- } \\
\text { Fibra } \\
\text { (kg/ha) }\end{array}$ & $\begin{array}{c}\text { Peso de } \\
\text { mota } \\
(\mathbf{g})\end{array}$ & $\begin{array}{c}\text { Longitud } \\
(\mathbf{m m})\end{array}$ & $\begin{array}{c}\text { Uniformidad } \\
\text { (\%) }\end{array}$ & $\begin{array}{c}\text { Resistencia } \\
\text { (gr/tex) }\end{array}$ & $\begin{array}{c}\text { Finura } \\
\text { (mg/pulg) }\end{array}$ \\
\hline L. Cesar -149 & 3.329 & 40,44 & 1.329 & 5,16 & 1,175 & 77,54 & 28,40 & 4,20 \\
\hline L. Cesar -151 & 3.490 & 42,19 & 1.461 & 5,30 & 1,174 & 77,46 & 28,23 & 4,29 \\
\hline L. Cesar -153 & 3.030 & 41,45 & 1.236 & 5,15 & 1,181 & 80,56 & 28,92 & 4,44 \\
\hline L. Turipaná 1 & 3.308 & 36,51 & 1.187 & 5,74 & 1,149 & 79,25 & 27,79 & 4,34 \\
\hline L. Turipaná 2 & 3.196 & 35,65 & 1.125 & 5,80 & 1,133 & 79,69 & 26,67 & 4,40 \\
\hline L. Turipaná 3 & 3.146 & 36,75 & 1.109 & 5,52 & 1,142 & 80,03 & 27,62 & 4,33 \\
\hline L. Turipaná 4 & 3.300 & 37,91 & 1.231 & 5,56 & 1,131 & 79,52 & 26,84 & 4,45 \\
\hline Corpoica M-123 & 3.176 & 39,88 & 1.256 & 5,38 & 1,168 & 80,38 & 28,11 & 4,51 \\
\hline Delta Opal & 3.615 & 38,63 & 1.379 & 5,45 & 1,157 & 81,99 & 29,32 & 4,55 \\
\hline Sinuana M-137 & 3.295 & 40,34 & 1.315 & 5,22 & 1,136 & 81,27 & 28,35 & 4,36 \\
\hline
\end{tabular}


mayor longitud de fibra con relación a las variedades comerciales y atributos de uniformidad, resistencia y finura, que satisfacen los estándares de calidad de fibra requeridos por la industria textil.

El análisis de adaptabilidad y de estabilidad con la metodología de Lin E Binns (1988a) resalta la inestabilidad de los cultivares L. Turipaná 1; L. Turipaná 2, L. Turipaná 3 y L. Turipaná 4 oriundos del departamento del Córdoba, por los altos valores de Pig. De igual manera, acusaron bajos rendimientos de fibra y de longitud de fibra (Tabla 3), lo que corrobora lo anotado por Morais et al. (2001), quienes indican que la mayor estabilidad está obligatoriamente asociada a una mayor producción y viceversa. Teniendo en consideración que esta metodología se basa en una comparación con relación a la mejor media de cada ambiente, es posible que estas líneas adolezcan de genes favorables para un mejor rendimiento de fibra y ellos contribuya a un mayor Pig.

La aplicación del método de Lin E Binns (1988a) modificado por Carneiro (1998), contenida en la Tabla 2, resalta como más estables para condiciones favorables Pif, los cultivares L. Cesar -151 y Delta Opal, que ocuparon el primero y segundo lugar de la clasificación, con Pif de 68,43 y 4904,0, respectivamente; siendo mucho mejor L. Cesar -151 que la variedad comercial. En condiciones ambientales desfavorables, se destacó como la más estable la línea L. Cesar -149, con un Pid de 6103,99, seguida de Corpoica $M$ - 123, con 8714,42 y Delta opal con 9438,54 . Estos resultados señalan la posibilidad de entregar a los agricultores nuevos materiales con ventajas comparativas en su estabilidad y en su rendimiento de fibra en condiciones desfavorables.

Como se aprecia en la Tabla 2, los métodos de análisis aplicados no arrojaron resultados muy discrepantes en la conducta de los genotipos estudiados y sí complementarios para una mejor decisión por parte de CORPOICA en la discriminación genotípica de sus líneas. Además, son métodos simples y eficientes para estimar en un solo parámetro la adaptabilidad y la estabilidad y su relación con el rendimiento de fibra, para su posterior recomendación a las áreas productoras.

Los índices de adaptabilidad y de estabilidad para localidades Pil están contenidos en la Tabla 4. Se observa que, en términos generales, hubo una mejor oferta ambiental con relación a fertilidad de suelos, precipitación y manejo agronómico en el caribe húmedo

Tabla 4. Rendimiento de fibra (kg/ha-1) de diez cultivares de algodón evaluados en ocho ambientes del caribe colombiano, 2003-2004

\begin{tabular}{|c|c|c|c|c|c|c|c|c|c|}
\hline \multicolumn{10}{|c|}{ LOCALIDADES } \\
\hline Cultivares & Maícao & El Copey & $\begin{array}{c}\text { Codazzi } \\
\text { (Motilonia) }\end{array}$ & $\begin{array}{c}\text { San Juan del. } \\
\text { Cesar }\end{array}$ & $\begin{array}{c}\text { Cereté } \\
\text { (Turipaná) }\end{array}$ & San Pelayo & Cotorra & Aguachica & $x$ varietal \\
\hline LC- 149 & 1433 & 817 & 737 & 999 & 1615 & 1741 & 1915 & 1376 & 1329 \\
\hline LC- 151 & 1690 & 895 & 637 & 986 & 1786 & 2007 & 2202 & 1486 & 1461 \\
\hline LC- 153 & 1550 & 800 & 619 & 832 & 1759 & 1441 & 1540 & 1346 & 1236 \\
\hline LT- 1 & 1530 & 643 & 692 & 732 & 1509 & 1592 & 1584 & 1219 & 1188 \\
\hline LT- 2 & 1262 & 457 & 652 & 941 & 1401 & 1546 & 1665 & 1075 & 1125 \\
\hline LT- 3 & 1299 & 529 & 636 & 947 & 1369 & 1471 & 1418 & 1207 & 1110 \\
\hline LT- 4 & 1551 & 679 & 721 & 852 & 1532 & 1567 & 1587 & 1360 & 1231 \\
\hline CM-123 & 1532 & 755 & 731 & 1011 & 1749 & 1319 & 1749 & 1206 & 1257 \\
\hline DP opal & 1592 & 765 & 911 & 812 & 1664 & 1778 & 2113 & 1399 & 1379 \\
\hline SM- 137 & 1577 & 780 & 715 & 957 & 1812 & 1485 & 1857 & 1340 & 1316 \\
\hline$x$ localidad & 1501 & 712 & 705 & 907 & 1619 & 1594 & 1763 & 1301 & \\
\hline Pil & 4290 & 69037 & 69960 & 45796 & 1296 & 1785 & 0 & 13340 & \\
\hline
\end{tabular}


respecto al caribe seco, a excepción de Maicao, ya que en estas localidades se registró un mayor rendimiento de fibra/ha, lo que corrobora lo expresado por Chloupek E Hrstkova (2005). Morais et al. (2001), resaltan la correlación entre la oferta ambiental y el rendimiento. Se destacaron por su rendimiento de fibra superior a $1500 \mathrm{~kg} / \mathrm{ha}^{-1}$ en el caribe húmedo Cotorra, Cereté y San Pelayo, en tanto que en el caribe seco, Maicao. Sobresalen, como los depeor oferta ambiental, El Copey y Codazzi (C.I. Motilonia), que hace de estas áreas del caribe seco marginales para el cultivo del algodón con estos genotipos, por la alteración de las lluvias, pérdida paulatina de la fertilidad de suelos e irregularidad en el manejo de problemas fitosanitarios.

La respuesta diferencial de los cultivares a la oferta ambiental del caribe colombiano permite separar los ambientes y recomendar los cultivares con mayor adaptación específica para una región en particular y así aprovechar las condiciones ambientales previsibles, como sugieren Lin \& Binns (1988b) y tecnológicas para una mejor producción Chloupek et al. (2004).

\section{CONCLUSIONES}

Los cultivares difieren para rendimiento de fibra en su comportamiento en cuanto a la adaptabilidad y la estabilidad fenotípica, con posición privilegiada para las líneas promisorias L. Cesar -151 y L. Cesar -149, que ocuparon el primer lugar en la clasificación por estabilidad, por rendimiento de fibra y por calidad de la misma en condiciones ambientales favorables $\mathrm{y}$ desfavorables.

El caribe húmedo posee mejor oferta ambiental para la expresión genética de los cultivares de algodón y, por lo tanto, mejor competitividad de sus productores.

\section{BIBLIOGRAFÍA}

ABEYSIRIWADERNA, D.S. 2001.Statistical analysis of on-farm yield trials for testing adaptability of rice. Euphytica. 121(3):215-222.

ARIAS, A.F. 2007. El sector algodonero en Colombia. Ministerio de Agricultura y Desarrollo Rural. Cereté, Mayo 25 de 2007.
ATROCH, A.L.; SOARES, A.A.; PATTO, M.A. 2000. Adaptabilidade e estabilidade de linhagens de arroz de sequeiro testadas no estado de Minas Gerais. Ciência e Agrotecnologia. 24(3):541-548.

BANZIGER, M.; COOPER, M. 2001. Breeding for low input conditions and consequences for participatory plant breeding: examples from tropical maize and wheat. Euphytica. 122(3):503-510.

CARNEIRO, P.C.S. 1998. Novas metodologias de análise da adaptabilidade e estabilidade de comportamento. Viçosa, Tese (Doutorado em Genética e Melhoramento). Universidade Federal de Viçosa. $155 p$.

CHLOUPEK, O.; HRSTKOVA, P. 2005. Adaptation of crops to environment. Theoret. Appl. Genetics.111(7):1316-1321.

CHLOUPEK, O.; HRSTKOVA, P.; SCHWEIGERT, P. 2004. Yield and its stability, crops, diversity, adaptability and response to climate changes, weather and fertilization over 75 years in the Czech Republic in comparison to some European countries. Field Crops Res. 85(2-3):167-190.

CRUZ, C.D.; REGAZZI, A.J. 1997. Modelos biométricos aplicados ao melhoramento genético. 2.ed. Viçosa: UFV. 390p.

DENCIC, S.; KASTORI, R.; KOBILJSKI, B.; DUGGAN, B. 2000. Evaluation of grain yield and its components in wheat cultivars and landraces under near optimal and drought conditions. Euphytica. 113(1):43-52.

DUARTE, J.B.; VENCOVSKY, R. 1999. Interação genótipos $\mathrm{x}$ ambientes: uma introdução à análise. "AMMI". Riberão Preto: Sociedade Brasilera de Genética. (Monografias, 9). 60p.

EBERHART, S.A.; RUSSELL, W.A. 1966. Stability parameters for comparing varieties. Crop Science. 6(1):36-40.

ESPINAL, C.F.; COVALDA, H.M.; PINZÓN, N.; BARRIOS, C.A. 2005. La cadena de algodón en Colombia 
una mirada global de su estructura y dinámica 1991-1995. Ministerio de Agricultura y Desarrollo Rural. Observatorio Agrocadenas Colombia. Documento de Trabajo N$^{\circ} 90$. Disponible desde Internet en: http://www.agrocadenas.gov.co. (con acceso 20/03/07).

ESPITIA, M.; ARAMENDIZ, H.; MENDOZA, A. 1993. Selección simultánea de genotipos de algodón (Gossypium hirsutum L.) por altos rendimientos y estabilidad. Revista ICA. 28(3):227-234.

FERREIRA, R. de P.; BOTREL, M. de A.; RUGGIERI, A.C.; PEREIRA, A.V.; FERNANDES, A.D.; DA SILVA, F.J.; CRUZ, C.D. 2004. Adaptabilidade e estabilidade de cultivares de alfafa em relação a diferentes épocas de corte. Ciencia Rural. 34(1):265-269.

FINLAY, K.W.; WILKINSON, G.N. 1963. The analysis of adaptation in a plant breeding program. Australian J. Agric. Res. 14:742-754.

GAUCH, H. 1988. Model selection and validation for yield trials with interaction. Biometrics. 44:705-715.

GAUCH, H.; FURNAS, R. 1991. Statistical analysis of yield trials matmodel. Agron. J. 83(4):916-920.

HUEHN, M. 1990. Nonparametric measures of phenotypic stability. Part 1: Theory. Euphytica. 47(3):189-194.

JACOBSEN, S.E.; HILL, J.; STOLEN, O. 1996. Stability of quantitative traits in quinoa (Chenopodium quinoa Willd). Theoret. Appl. Genetics. 93(12):110-116.

KANG, M.S. 1998. Using genotype-by-environment interaction for crop cultivar development. Advanc. Agronomy. 35:199-240.

KATO, K.; MIURA, H.; SAWADA, S. 2000. Mapping QTLs controlling grain yield and its components on chromosome 5 A of wheat. Theoret. Appl. Genetics. 101(7):1114-1121.

KROONENBERG, P.M.; BASFORD, K.E. 1989. An investigation of multiattribute genotype response across environments using three mode principal component anlysis. Euphytica. 44(1-2):109-123.
LIN, C.S.; BINNS, M.R. 1988a. A superioriorite measure of cultivar performance for cultivar x location data. Can. J. Plant Science. 68(2):193-198.

LIN, C.S.; BINNS, M.R. 1988b. A method of analyzing cultivar $\mathrm{x}$ location $\mathrm{x}$ year experiments: a new stability parameter. Theoret. Appl. Genetics. 76(3):425-430.

LIN, C.S.; BUTLER, G. 1990. Cluster analysis for analyzing two-way classification data. Agron. J. 82(2):344-348.

LIN, C.S.; BINNS, M.R.; LEVKOVITCH, L.P. 1986. Stability analysis: where do we stand? Crop Science. 26(5):894-900.

LIN, C.S.; THOMPSON, B. 1975. An empirical method of grouping genotypes based on a linear function of the genotype-environment interaction. Heredity. 34:255-263.

MENDOZA, A.; ARAMENDIZ, H.; URREA, R. 1999. Origen, características y comportamiento productivo de nuevas líneas de algodón para el caribe húmedo. En: Memorias Curso Manejo del Algodonero, Plan Nacional de Algodón, Corpoica - Conalgodón - Fondo de Fomento Algodonero. p.13-17.

MORAIS, S.A.; AZEVEDO FILHO, J.A.; DOS SANTOS DIAS, L.A.; GONÇALVES, CH.; BIANCHINI, C. 2001. Adaptabilidade e estabilidade de produção de cultivares e linhagens de feijoeiro no estado de São Paulo. Bragantia. 60(2):69-77.

MURAKAMI, D.M.; CARDOSO, A.A.; CRUZ, C.D.; BIZAO, N. 2004. Considerações sobre duas metodologias de análise de estabilidade e adaptabilidade. Ciência Rural. 34(1):71-78.

PLAISTED, R.L. 1960. A shorter method of evaluating the ability of selection to yield consistently over seasons. Am. Potato J. 37:166-172.

PIEPHO, H.P. 1996. Analysis of genotype by environment interaction and phenotypic stability. In: Kang, M.S.; Gauch Jr., H.G. (Eds) Genotype by interaction. CRC Press, Boca Ratón, Florida. p.151-174. 
SAS INSTITUTE, 2002. Software and Services: System for windows, version. 8.0

SCAPIM, C.A.; RODRIGUES, V.; BRACCINI, A.; CRUZ, C.D.; BASTOS ANDRADE, C.A.; GONÇALVES, M.C. 2000. Yield stability in maize (Zea mays L.) and correlations among the parameters of the Ebehart and Russell, Linn and Binns and Huehn models. Genetics and Molecular Biology. 23(2):387-393.

SHUKLA, G.K. 1972. Some statistical aspects of partitioning genotype - environmental components of variability. Heredity. 29:237-245.

SNAPE, J.W.; FOULKES, M.J.; SIMMONDS, J.; LEVERINGTON, M.; FISH, L.J.; WANG, Y.; CIAVARRELLA, M. 2007. Dissecting gene $x$ environmental effects on wheat yield via QTL and physiological analysis. Euphytica. 154(3):401-408.
SOUZA, D.N.A.; HOLANDA, D.J.S. 1993. Adaptabilidade ambiental de cultivares de algodoeiro mocó. Pesquisa Agropec. Bras. 28(7):797-801.

VERMA, M.M.; CHAHAL, G.S.; MURTY, B.R. 1978. Limitations of conventional regression analysis: a proposed modification. Theoret. Appl. Genetics. 53(2):89-91.

VERTEL, M.; ESPITIA, M.; MARTÍNEZ. R. 1999. Comparación de ocho índices para determinar estabilidad fenotípica en algodón. Temas Agrarios. 8:69-80.

WRICKE, G. 1962. Über eine Methode zur Erfassung der ökologischen Streubreite in Feldversuchen. Z. Pflanzenzücht. 47(1):92-96.

Recibido: abril 9 de 2007

Aceptado: septiembre 19 de 2007 